EDUCATION

\title{
Complex systems model of fatigue: integrative homoeostatic control of peripheral physiological systems during exercise in humans
}

\author{
E V Lambert, A St Clair Gibson, T D Noakes
}

Br J Sports Med 2005;39:52-62. doi: 10.1136/bjsm.2003.011247

Fatigue is hypothesised as being the result of the complex interaction of multiple peripheral physiological systems and the brain. In this new model, all changes in peripheral physiological systems such as substrate depletion or metabolite accumulation act as afferent signallers which modulate control processes in the brain in a dynamic, nonlinear, integrative manner.

See end of article for authors' affiliations

Correspondence to: Associate Professor Lambert, University of Cape Town, Research Unit for Exercise Science and Sports Medicine, Sports Science of South Africa, PO Box 115, Newlands 7725 , South Africa; vlambert@sports.uct.ac.za

Accepted 8 June 2004
$\mathrm{T}$ o a large degree, our understanding of the aetiology and underlying mechanisms of fatigue during exercise has been influenced by a reductionist approach, despite over 3000 scientific publications on this topic within the past 20 years. This approach may best be illustrated by relating the well known Indian fable of the blind men and the elephant. The first blind man, grasping the ear of the elephant, remarked that an elephant was very much like the sail of a ship. The second blind man, holding the leg of the creature, compared the elephant with the trunk of a tree. The third blind man, clutching the trunk, argued that an elephant was similar to a snake, and the fourth blind man, grasping the tail, considered all of his fellows to be incorrect, because it was very clear that an elephant was like a thick piece of rope.

Using a reductionist approach, all of these men were correct, in part. The elephant is like all, yet like none, of these individual observations. However, it is only by "seeing" the entire elephant that one can explain the individual contribution of each of these parts to the whole. This is not unlike the study of fatigue during exercise, typically undertaken within a fragmented and teleological framework. Teleology is the appearance of having been designed for a purpose. However, the teleological explanation for fatigue depends entirely on one's perspective, or to put it another way, "what part of the elephant one is holding". This approach has led to the development of the peripheral model of fatigue, which posits that system failure is the consequence of, for example, metabolite accumulation or substrate depletion, and system failure can only be averted by a reduction in workload or the termination of exercise.

Noakes and St Clair Gibson ${ }^{1}$ have presented evidence that fatigue is dissociated from ATP depletion, and that ATP depletion does not occur during either high intensity exercise of short duration or during more prolonged exercise. St
Clair Gibson and Noakes ${ }^{2}$ have further suggested that there is central control of exercise intensity, based on some "black box calculation" or neural integration of afferent information from the periphery, influenced by such factors as prior experience and training. They suggest that this neural integration leads to an oscillatory power output and physiological responses during exercise, the result of complex integration of inputs from multiple systems, and including both feed forward and feedback components. St Clair Gibson and Noakes ${ }^{2}$ have further argued that fatigue is a sensory perception, rather than a physical phenomenon. It is the purpose of this review to extend this theory by presenting evidence for a complex, but centrally integrated model of fatigue, and to examine candidates from the periphery that may provide afferent feedback for this black box calculation, informing the perception of fatigue and resulting in an attenuation of work output, which may be consciously or unconsciously determined.

An integrated model of fatigue may be considered in the context of the homoeostatic control of central and peripheral physiological systems. A "homoeostat" is a negative feedback controller, which is necessary for the maintenance of life. Examples of homoeostats include arterial oxygen saturation level, blood glucose concentration, acid-base balance, arterial blood pressure, core temperature, and plasma osmolality. ${ }^{3}$ In effect, extreme changes in any of these homoeostats are incompatible with life, and as such, must be protected against, as part of an active neural process, ${ }^{2}$ during so called "maximal" exercise, the function of which may be to prevent the development of absolute fatigue and consequent organ damage. An example of failure of these homoeostatic control mechanisms is seen in malignant hyperthermia. With the use of centrally active drugs such as halothane, anaesthesia induces a malignant increase in temperature, which leads to heat induced myopathy, renal failure, and death. This disease process does not affect the individual during activities of daily living, and is induced by the anaesthetic drugs over-riding the active neural processes associated with temperature regulation in the central nervous system.

Therefore these active neural processes ensure that homoeostats are maintained by preset,

Abbreviations: EMG, electromyography; MSNA, muscle sympathetic nerve activity; MVC, maximal voluntary contraction; RPE, rating of perceived exertion 
programmed responses and feed forward control. "Gain" is the extent to which a particular system deviates from baseline after having responded to a perturbation. Therefore a "perfect homoeostat" is defined as having infinite gain, which is achieved through integral control. However, as homoeostasis must be maintained or restored in many physiological systems during exercise, the gain of a particular homoeostat may be increased through multiple, often opposing, but interdependent controllers. ${ }^{4}$ This may be referred to as integrative control. Furthermore, the time constant of the gain of a homoeostat refers to how quickly a system returns to baseline after a perturbation. The time constant is mutable, and may have implications for homoeostatic control of interlinked systems, as a delay in one may alter the gain of another. In this review, we will attempt to relate integrative control of homoeostatic processes to motor drive and the perception of fatigue. Moreover, we will attempt to advance the argument that training and other antecedent stimuli result in changes in both integrative homoeostatic control and central programming, altering the settings at which gain is optimised, and thereby informing and governing the perception of fatigue.

\section{THE BLACK BOX CALCULATION OF MUSCLE METABOLIC RATE: EVIDENCE FOR REGULATION OF PACING DURING PHYSICAL ACTIVITY}

In 1996, Ulmer ${ }^{5}$ proposed a hypothetical model of a control system for optimisation of "performance" during heavy exercise. The model is based on a standard feedback control loop, in which the efferent signals contain information on motion, force output, time, and muscular metabolism, thereby determining muscle metabolic rate and exercise intensity. Afferent signals then feed back information from chemoreceptors and mechanoreceptors which may be used to alter or modify movement and force output to "optimise" performance.

However, Ulmer ${ }^{5}$ also suggested that the model was more complex, and that afferent feedback may arise from muscle (somatosensory) or peripheral organs or both, that there may be endogenous reference signals, and that factors such as training, muscle reserve, muscle metabolic rate, and prior or antecedent experiences may alter the interpretation of this afferent input (fig 1). According to Ulmer, ${ }^{5}$ these endogenous reference signals are found in a central "black box" programmer, which also takes into account projected "finishing points" when calculating later efferent commands based on afferent input of metabolic activity. Subsequent muscle output is modified as a result of this integrative control. He referred to this integrated model as teleoanticipation.

Teleoanticipation also differs from the better known concept of feed forward control. Feed forward control strategies, which can also be described as anticipatory responses, involve a large element of guess work. An example of feed forward control is the donning of warm clothing before venturing out of a house, after having seen an overcast sky and the wind through a window while one is still warm inside the house. Whether enough or too much clothing has been put on will only become apparent when the skin temperature receptors are exposed to the outside air. At this point feedback control occurs, which is responsible for any corrective response, and is not based on guess work but is a true homoeostatic response $(\mathrm{J} H$ Koeslag, personal communication).

The model of teleoanticipation is specific for exercise and associates programming of power output and pacing strategies with the end point of the exercise bout. Teleoanticipation is less dependent on guess work than feed forward responses, as it includes both feed forward planning and feedback control from afferent changes associated with peripheral metabolic structures and the external environment, and also incorporates knowledge acquired from prior exercise bouts. Although there is still an element of guess work required to adjust the exercise rate in the hopes of arriving at the end before catastrophic fatigue prematurely limits the exercise performance, this is far less than would occur with either a simple feed forward control or a simple feedback mechanism.

To test the model, Ulmer ${ }^{5}$ conducted a series of experiments in which athletes were asked to run $400 \mathrm{~m}$ and $1500 \mathrm{~m}$ on two separate occasions. For each distance, athletes were instructed to run maximally or at specific levels of submaximal effort perception (rating of perceived exertion (RPE)), using the Borg scale. Although running velocities or performances were graduated according to RPE, in the $1500 \mathrm{~m}$ distance, even when athletes were asked to run maximally, the pace or running velocity at each RPE level between the distances of $400 \mathrm{~m}$ and $1500 \mathrm{~m}$ remained remarkably constant and submaximal. These results suggest that, over longer distances, running velocity is preset and that pacing or teleoanticipation occurs, even at so called maximal exercise, in order to reach a predetermined finish point.

Hampson et $a l^{6}$ further explored the concept of teleoanticipation by introducing a deliberate mismatch in actual and expected exercise intensity and by documenting the perception of effort in response to this deception. Forty well trained runners were randomised to one of two trials. In the first trial, the subjects ran at one of three intensities $(80 \%, 83 \%$, or $86 \%$ of peak treadmill running speed) on an indoor track using pacing lights. The order of these runs was randomised, and the experimental group was told that all runs were at the same intensity, $83 \%$ of peak treadmill running speed. In trial 2 , subjects ran at $83 \%$ of peak treadmill running speed for all three runs, but the experimental group was told that the runs were of increasing intensity $(80 \%, 83 \%$, and $86 \%$ of peak treadmill running speed).

Runners who were deceived by being told that exercise intensity would be held constant had similar increases in their overall perception of effort (RPE) to a control group with subsequent exercise bouts of increasing intensity. This suggests that the subconscious interpretation of afferent feedback, monitoring exercise intensity, was correct, despite being provided with deliberate misinformation. Thus, in this example, the subconscious interpretation of exercise intensity is either independent of, or influenced to a lesser extent by, prior knowledge or programming.

Conversely, during consecutive runs at a constant intensity, control runners showed an order effect, reporting increased RPE with each subsequent exercise bouts. However, subjects in the experimental group, who were expecting consecutive runs of increasing work intensity did not report any increase in RPE of the chest and leg regions with subsequent runs. This suggests that, at a conscious or subconscious level, subjects misinterpreted the afferent input, which resulted in an increased RPE in the control group, perhaps as a consequence of the work intensity being less than expected. Thus anticipation based on prior experience or "forecasting" may alter the perception of fatigue.

Hampson et $a l^{7}$ therefore suggested that there is a subconscious setting of exercise intensity based on prior experience of what is required to complete an exercise bout within the biomechanical and metabolic constraints of the body. This results in programming of efferent control signals. However, afferent feedback from the periphery (skeletal muscle and other organ systems) is then interpreted by the central nervous system against expected outcomes, resulting in the conscious perception of effort. Antecedent exposures, such as training, diet, or specific environmental feedback, may alter the interpretation of perception of effort, and may 

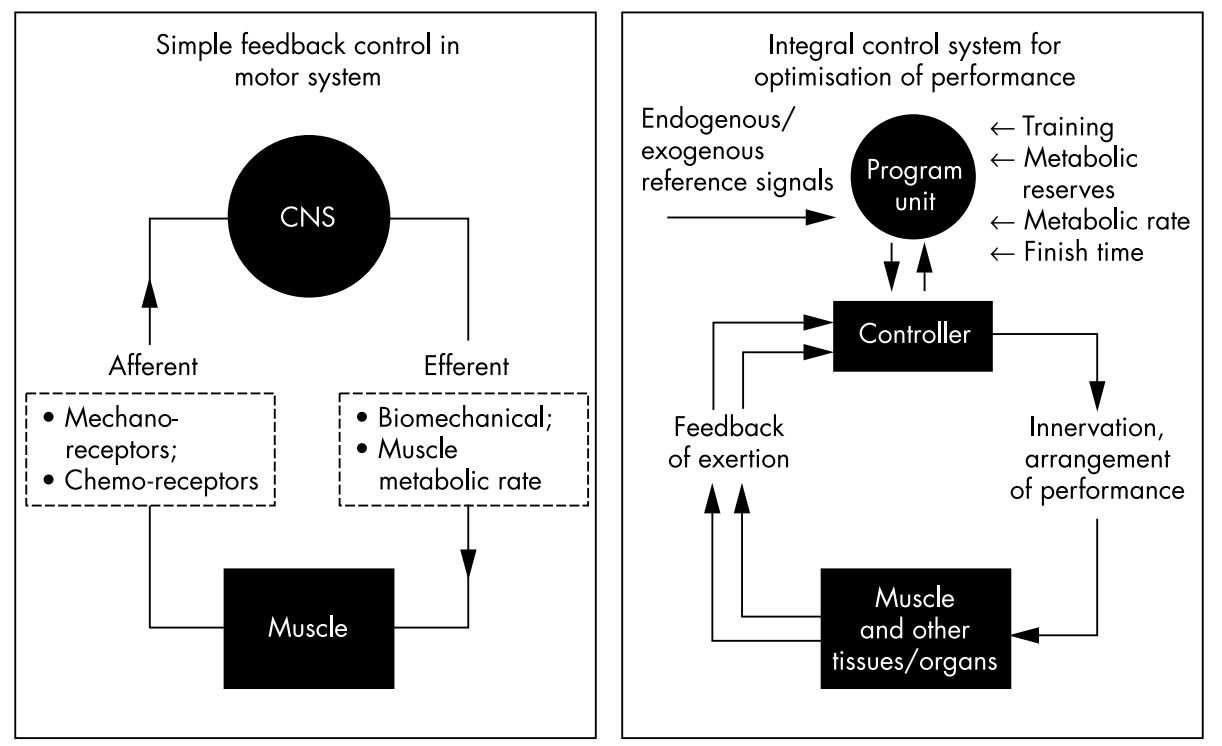

Figure 1 Ulmer's hypothetical model of a control system for optimisation of performance during heavy exercise. The left panel shows simple feedback control of the motor system by the central nervous system (CNS). The right panel shows an integrative control system for optimisation of physical performance where there are a number of different levels of control in the central nervous system and peripheral physiological systems. Reproduced, with permission, from Ulmer. ${ }^{5}$

then result in resetting of exercise intensity and the teleoanticipatory set points (fig 2).

Further evidence for an effect of teleoanticipation in the control of pacing strategies may be found in a study of effort perception during exercise in the heat. Nybo and Nielsen ${ }^{8}$ studied the relation between RPE, electromyography (EMG), and electroencephalography during submaximal exercise in normal and hot conditions, in endurance trained persons. They found that RPE, cerebral artery blood flow, and electroencephalographic changes, specifically the index of activation of the alpha relative to the beta waves, were all strongly and significantly related to core temperature during exercise. Moreover, RPE was unrelated to any measures of muscle recruitment. Indeed, muscle EMG amplitude and frequency remained unchanged during exercise in the heat. These data suggest that hyperthermia induced "fatigue" may be a consequence of central programming, which is associated with a progressively increasing perception of fatigue during exercise in the heat, even though power output and muscle recruitment do not change, and this central programming change may be activated by awareness of rate of change in core temperature, heat storage, or other metabolic variables.

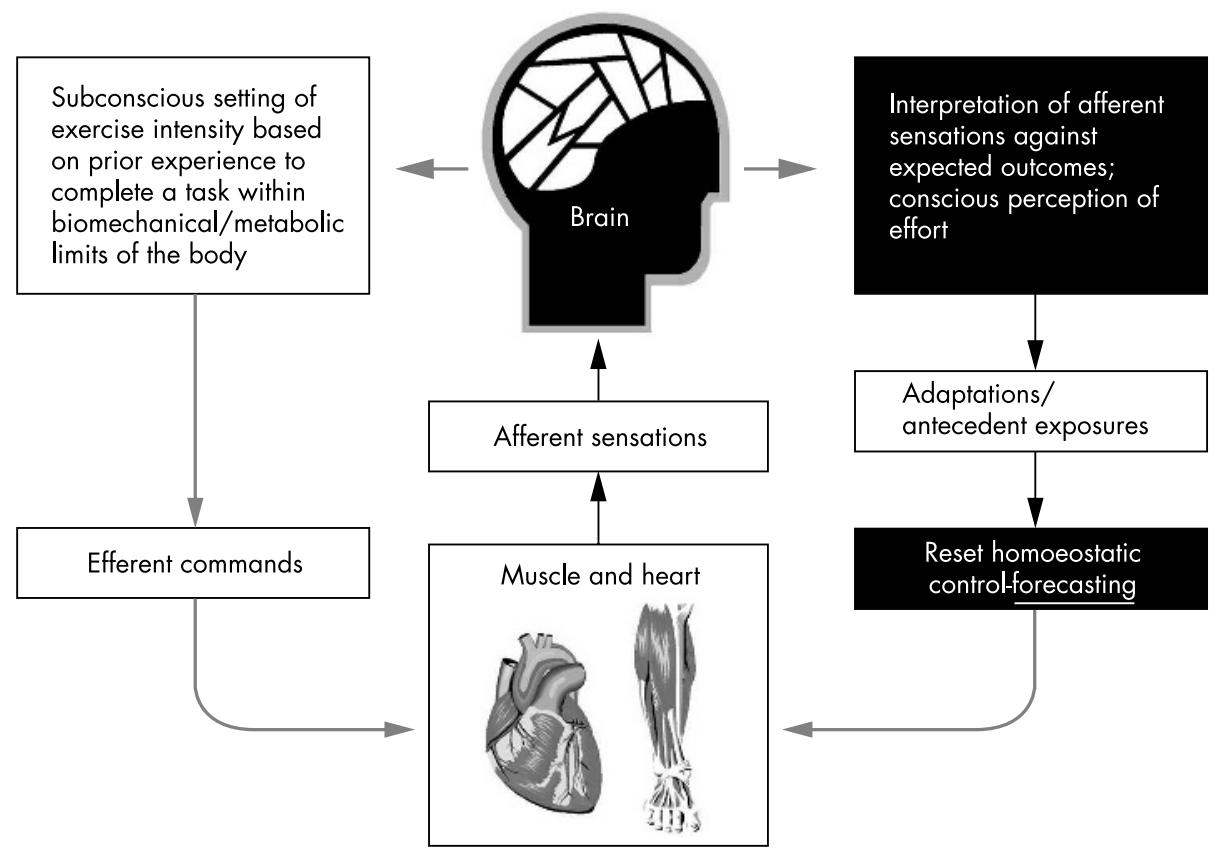

Figure 2 Relation between the teleoanticipatory governor centre in the brain and perceived exertion during exercise. Exercise intensity is set at a subconscious level by the central nervous system, and the perceived effort is the interpretation of afferent sensations against expected outcomes set by the subconscious teleoanticipatory governor centre. 
Perhaps one of the most provocative examples of performance optimisation by teleoanticipatory pacing strategies and feedback control is that of the long distance flights of migratory birds. These birds require large "on-board" fuel stores at the outset of their journeys, to cover non-stop flight distances over several thousand kilometres. Remarkably, metabolic fuel is converted into mechanical power output most efficiently at the outset of the journey, when fuel loads are greatest, despite the greater mechanical power output required to fly at heavier weights. This change in efficiency seems to occur as the result of an increase in flight muscle size relative to the fuel stores (and hence body mass), at the expense of manoeuvrability. Thus, in migratory birds travelling non-stop distances of more than $4000 \mathrm{~km}$, pacing occurs in the form of increased metabolic efficiency, or a greater sparing of fuel stores, at the outset of the journey, when mechanical power output is greatest because of the increased mass or fuel load.' Similarly, changes in mechanical efficiency related to distance to be covered during a running trial have also been found in humans. In a recent study performed in our laboratory, athletes who were unaware of the distance to be covered during the trial ran with greater mechanical efficiency than when they knew that they were running for a set time of 10 or 20 minutes (W Baden, $\mathrm{R}$ Tucker, I McLean, A St Clair Gibson, TD Noakes, unpublished data).

Further, other evidence suggests that changes in substrate selection from available fuel reserves and in response to the imposed stress during fasted migratory flight in birds are regulated by neurohormonal programming and feedback. ${ }^{10}$ For example, blood corticosteroid concentrations increase in migrating birds only when fat stores become depleted, and flight muscles are reduced in size as a result of muscle protein breakdown.

Birds may also have increased corticosteroid responses to stress associated with unpredictable events such as bad weather or human interference. Behaviours typically associated with this hormonal stress response are seeking shelter and increased food foraging. What is not known is whether increased corticosteroid concentrations in migrating birds cause a change in behaviour, resulting in landing early in order to forage for food, which would represent a change in the "finishing point". Perhaps sensory feedback on the size of fuel reserves, which may be influenced by other stressors, may alter the pacing strategies and teleoanticipation of these migratory birds, causing an early landing.

In summary, the linear model of fatigue suggests that exercise would continue until the muscle, for example, was completely depleted of substrate, or the system was overwhelmed by metabolite accumulation. In contrast, the central governor model suggests that metabolic variables are important "sensors" of change themselves and initiate afferent feedback to the brain, which in turn directly resets metabolic and motor activity in a feed forward manner. Efferent neural commands may regulate metabolic activity directly through neurometabolic pathways or indirectly by adjusting skeletal muscle motor unit recruitment with secondary changes in metabolic activity. The essential difference between these two models is that, in the teleoanticipation model, the outcome of changes in metabolic activity is to ensure that homoeostasis of the peripheral physiological systems is maintained, whereas in the linear model, metabolic changes occur in response to the attempt to maintain a specific exercise intensity. As described by St Clair Gibson and Noakes, ${ }^{2}$ fatigue in the linear model is the consequence of a catastrophic breakdown in system function, when metabolic demand overwhelms the capacity of peripheral tissues.

\section{THE EXERCISE "REFLEX" AND FEED FORWARD CONTROL OF THE NEUROMETABOLIC RESPONSE TO EXERCISE}

There is substantial in vivo and in situ evidence that metabolic activity during exercise is regulated in an integrated manner, incorporating both feed forward and feedback control. ${ }^{11-13}$ Vissing ${ }^{14}$ posited that the exercise reflex involves an "efferent mechanism associated with a simultaneous drive of the somatomotor and autonomic circuitry from cerebral motor centres, that in parallel stimulates (1) locomotion, (2) neuro-endocrine activity, (3) circulation and (4) an afferent mechanism associated with reflex stimulation of the working muscle".

In a novel series of experiments, Vissing ${ }^{14}$ systematically developed models for studying the neural control of this exercise or muscle reflex, in an attempt to localise the site of the central command process. In the first set of experiments, the posterior hypothalamic locomotor region, associated with the coordination of locomotion, was stimulated in intact, anaesthetised cats or in decorticated cats with neuromuscular blockade. ${ }^{15}$ This model prevented potential feedback from contracting muscles. Stimulation of the posterior hypothalamic locomotor region resulted in a direct increase in glucose production and a general state of sympathetic activation. These findings suggest that the activation of hepatic glucose output may result primarily from feed forward stimulation originating from the posterior hypothalamic locomotor region and is perhaps unrelated to the actual exercise intensity or muscle metabolic rate.

Vissing also examined the afferent components of this control process, using a "low force" muscle stimulation model to examine the impact of "movement" or mechanoreceptor feedback on the exercise reflex. ${ }^{14}$ Stimulation of the tibial motor nerve at twice the motor threshold in rats with and without neuromuscular blockade produced an increased sympathetic activation of the adrenal medulla. These data suggest that sympathetic activation of the adrenal medulla during exercise is also mediated by a mechanoreflex originating from contracting muscles, and is not solely a component of a humoral metaboreflex.

Further evidence for feed forward control was provided by a model in which the femoral nerve was cut in anaesthetised cats. ${ }^{14}$ The proximal part of the cut motor branch was then stimulated at 3,20, and 140 times the motor threshold under neuromuscular blockade to prevent humoral feedback from the hindlimb muscles. Only higher levels of stimulation activated group III and group IV afferents. Activation of these afferents produced a 2-5-fold increase in hepatic glucose production, plasma adrenocorticophic hormone concentrations, and heart rate. This increased hepatic glucose production has been referred to as "luxury glucose", as it is produced as part of the reflex response and is again unrelated to the actual metabolic load or metabolic requirement. ${ }^{14}$

Taken together, these data suggest that there is both mechanoreceptor and metaboreceptor stimulation of the exercise reflex, perhaps initiated at the onset of exercise by mechanical feedback and adjusted by chemoreceptor feedback according to the continuing energy needs of the muscle. Therefore these studies provide support for a model of integrative control of homoeostasis. Further, they suggest that the motor centres of the brain may initiate the exercise reflex; and that this reflex involves the feed forward activation of glucose counter-regulation and sympathetic activation.

There are likely to be redundancies in these control mechanisms. There is also likely to be a "fast component" sensitive to mechanoreceptor feedback, and a slower component, related to activation of chemosensitive receptors. However, in all these examples, the experimental animals 
were anaesthetised and underwent neuromuscular blockade. Therefore, although these researchers have effectively identified putative sites for central control of metabolic activity during exercise, the models do not permit study of the possible role of peripheral humoral feedback. There was also no integration of peripheral feedback, no nociceptive input, and no possible adaptation or teleoanticipation. Furthermore, the putative chemoactivators of this reflex are, as yet, undefined. In addition, the role of the activation of the muscle reflex in the perception of fatigue is not well understood.

However, some insights into neural activation and fatigue may be gained from studies of sympathetic nervous system activation measured with muscle sympathetic nerve activity (MSNA) and EMG activity during fatiguing, isometric exercise. ${ }^{16-20}$ Seals and Enoka ${ }^{16}$ studied efferent sympathetic neural discharge and EMG activity during repetitive bouts of fatiguing isometric handgrip exercise at $30 \%$ of the maximal voluntary contraction (MVC). They found that MSNA in noncontracting skeletal muscles in the leg correlated highly with EMG activity during exercise, but only after the initial 3090 seconds. For each of three successive bouts of activity, MSNA increased linearly throughout the exercise bout; EMG activity at any absolute time point after the first minute was increased, and time to fatigue was decreased. These data suggest that muscle recruitment for the same task was initially preset but altered for the same absolute work rate as a consequence of increased sympathetic activation. Investigators suggest that this link may be caused by chemoactivation of group III and IV afferents as a consequence of decreased perfusion associated with isometric contraction. In addition, they suggest that sympathetic activation may contribute to force generation by direct stimulation of contracting skeletal muscle with changes in skeletal muscle contractility.

More recently, however, Victor et $a l^{18}$ found that, during intermittent, submaximal handgrip exercise, MSNA was linked to the force of muscle contraction. There were no detectable increases in MSNA for intermittent isometric handgrip contractions at $25 \%$ and $50 \%$ MVC, but large increases at $75 \%$ MVC. Partial neuromuscular blockade with curare infusion resulted in a reduction in force output to less than $10 \%$ of MVC, but MSNA was not different from that during the $75 \%$ MVC bout.

These studies showed that intense, intermittent isometric exercise produced a synchronisation between motor activity and MSNA, such that each burst of motor activity was linked to one or more bursts of sympathetic nerve activity. It is likely that this synchronisation is caused, at least in part, by central command processes. This can be inferred from the finding that curarisation reduced force output to less than $10 \%$ of MVC, yet an "intent to exercise" alone was sufficient to produce an increase in MSNA similar to that measured during high intensity exercise. Furthermore, in a small subset of individuals, anaesthetic block of the axillary nerve resulted in a further increase in MSNA, suggesting that the effect of central command occurs even in the absence of muscle sensory feedback. Thus the intensity of contraction may preset the degree of muscle activation. However, central command processes, based on the "intent to exercise" and teleoanticipation of the workload cause increased sympathetic activation, even in the complete absence of muscle afferent feedback, providing further evidence for neural integration of the sensation of fatigue.

\section{REGULATION OF THE FATIGUE PROCESS: CANDIDATES FROM THE PERIPHERY}

As described previously, a linear model of fatigue assumes that peripheral metabolic demands exceed maximal capacity of the various systems, and that substrate depletion or metabolite accumulation is the direct cause of the onset of fatigue and associated termination of exercise. However, there is ample evidence that metabolic intermediates have an important role as signalling agents, regulating systems in an interactive, feedback manner rather than in a catastrophic system failure manner.

Therefore we propose that, in a complex model of fatigue, metabolic systems operate in a continuous and oscillatory fashion, and are governed such that these systems never operate at maximal capacity or to "failure". In this model, metabolic activity and both tissue specific and circulating metabolite and substrate concentrations have the capacity to signal afferent neural information to the central nervous system in a continuous manner in such a way that they are able to influence future adjustments to the exercise intensity or pace by the central governor. This model predicts that a number of different, or indeed all, peripheral physiological substrates and metabolites participate in the modulatory process, rather than the system being controlled by any single factor.

For example, recent studies have suggested that muscle glycogen content or the rate of muscle glycogenolysis may have afferent signalling properties. Weltan et al ${ }^{21}$ showed that low muscle glycogen concentrations, independent of associated hypoglycaemia, caused an exaggerated muscle reflex response with enhanced sympathetic activation and increased release of free fatty acids. They suggested that afferent sensory feedback from chemoreceptors in contracting muscle respond to either the low muscle glycogen concentrations or changes in concentrations of a glycolytic intermediate. They further suggested that this neural signalling is directed to the hypothalamic locomotor centres by type III and IV chemoreceptors, where the resulting metabolic changes are initiated..$^{21}$ These data suggest that the rate of muscle glycogenolysis or the muscle glycogen concentrations, or both, are critical for initiating changes in metabolic activity which would allow continuation of force output and energy expenditure and prevent system failure.

Re-analysis of a previous study from this unit ${ }^{22}$ adds further support to the existence of a "glycostat". In that study, subjects were asked to cycle for two hours at $75 \%$ $\mathrm{VO}_{2}$ peak, during which time they also completed five 60 second sprint bouts. Trial order was randomised, and subjects were either carbohydrate depleted or carbohydrate replete and ingested an $8 \%$ carbohydrate solution throughout the exercise bout. After completing the two hour exercise bout, subjects performed a one hour cycle time trial, during which they were blinded to all performance parameters except elapsed time.

The first important finding was that subjects achieved their highest work rates in the last few minutes of the time trial, when their muscle glycogen content would have been the lowest. This conflicts with the linear model of exercise physiology, ${ }^{2}$ which holds that muscle glycogen depletion impairs exercise performance. This ability to perform an end spurt also shows that skeletal muscle motor unit recruitment is submaximal during the earlier stages of the time trial. ${ }^{23}{ }^{24}$

The second key finding was that subjects performed significantly more work during the one hour time trial when they were glycogen replete, as is expected according to the predictions of either the linear or the central governor models. According to the linear model, the larger glycogen stores would allow more exercise to be performed before terminal muscle glycogen depletion and absolute fatigue developed. Alternatively, according to the teleoanticipation model, peripheral feedback from muscle chemoreceptors would inform the central governor that a greater exercise intensity or pace could be sustained for the anticipated 
duration of the trial, on the basis of existing fuel available at the start of the 60 minute time trial. This is similar in some sense to the information used by migratory birds before the beginning of migration. In this case, the fact that exercise intensity was greatest at the end of the trial when muscles were most glycogen depleted suggests that the complex, teleoanticipation model may provide a more likely explanation for pacing occurring during the time trial.

The third finding was that the difference in power output between trials was present within the first few minutes of exercise. This indicates that the pacing strategy was established in anticipation of and not only after metabolic failure developed.

Further support for this interpretation was the important finding of a remarkable intrasubject consistency in end exercise muscle glycogen concentrations, irrespective of total work done in the time trial and unrelated to starting muscle glycogen concentrations. In contrast, there was as much as a threefold difference in the end exercise muscle glycogen concentrations between subjects, indicating that each individual had a different critical "stopping" muscle glycogen concentration. However, it is likely that a similar signalling mechanism exists in all individuals, as the response is highly reproducible despite different starting muscle glycogen concentrations.

Taken together, these data are more compatible with a central governor model of fatigue during exercise in which the muscle glycogen concentration acts as a signal, rather than a metabolic determinant of absolute fatigue. ${ }^{21}$ This is in line with the putative role of the muscle glycogen concentration as a metabolic signal, either directly as in the case of glycogen binding, for example to the $\beta$ subunit of AMP kinase, $^{25}$ or in relation to the capacity of the muscle to respond to $\mathrm{T}$-system depolarisation, ${ }^{26}$ or indirectly, in association with increased release of interleukin 6 or adrenaline (epinephrine). ${ }^{27}$

Lactate is another example of a metabolite that may act as an important peripheral signalling agent rather than as a direct cause of catastrophic system failure as required in the linear, peripheral model, pioneered by the work of Hopkins, Fletcher, and Hill. ${ }^{1}$ More modern work has suggested that lactate is an important fuel source during high intensity exercise, ${ }^{28}$ rather than a simple metabolic byproduct, and that high lactate concentrations and associated acidosis may actually enhance rather than impair isolated skeletal muscle function in the presence of high extracellular $\mathrm{K}^{+} .{ }^{29}$ Lactic acid in that study counteracted the effects of high extracellular $\mathrm{K}^{+}$ concentrations associated with a decrease in force production, probably caused by inactivation of $\mathrm{Na}^{2+}$ channels. Thus lactic acid may protect against muscle fatigue during high intensity exercise, in which extracellular $\mathrm{K}^{+}$concentrations are also raised.

Further work has shown that lactic acid acts directly on acid-sensing ion channels which are highly expressed on sensory neurones so that, acting through afferent neural pathways to pain centres in the central nervous system, lactic acid may initiate sensations of pain associated with high intensity exercise. ${ }^{30}$ Therefore, apart from its role as an essential metabolic substrate rather than a metabolic byproduct, lactic acid may act as a signalling mechanism inducing feed forward, protective mechanisms such as reductions in exercise intensity, secondary to reduced motor unit recruitment by the central nervous system. Thus, lactic acid appears to have the potential for a regulatory role as part of the teleoanticipatory system described previously.

Glucose may also act as a potential metabolic signaller. Dalsgaard $e a^{31}$ calculated the ratio of oxygen to glucose and lactate uptake by the brain, by measuring the carotid arteryinternal jugular venous differences. The resting ratio is typically 6:1, but, immediately after maximal exercise, this ratio was reduced to 3.7:1. There was no change in the ratio after submaximal exercise. However, after subjects had exercised at submaximal levels under partial neuromuscular blockade, the ratio was significantly reduced from a resting ratio of $6: 1$ to $4.9: 1$ in recovery. In the same study, perceived exertion, heart rate, and mean arterial pressure were all higher under neuromuscular blockade, compared with control conditions, despite the fact that work rates were the same or lower. The authors concluded that central command, or, in this case, the "intent to exercise" maximally, even in the absence of appropriate metabolic feedback, may result in altered brain metabolism, perhaps signalling the sensation of fatigue.

There are other more obvious examples of peripheral metabolic signallers that have afferent input to the central nervous system and affect the function of different systems in an integrative manner. Plasma interleukin 6 concentrations increase during exercise, and have a direct afferent input to the brain, leading to the initiation of cognitive strategies resulting in a reduction in physical activity. ${ }^{32}$ Therefore this is an example of a metabolite that provides the signalling required for the central black box calculations that occur in the brain, predicted by the central governor model. As a result of these calculations, alterations in pacing strategy occur in a feedback regulatory manner.

Another likely candidate in the peripheral system that may act to modulate exercise intensity directly in relation to effort perception is intramuscular $\mathrm{Ca}^{2+}$ concentrations. $\mathrm{Ca}^{2+}$ is involved as part of a second messenger system in numerous metabolic pathways-for example, activating AMP kinase and initiating excitation-contraction coupling. ${ }^{33}$ A recent review ${ }^{34}$ summarised the possible sites and mechanisms of peripheral fatigue, including $\mathrm{Ca}^{2+}$, during repetitive muscle contractions, which represented endurance muscle activity. In numerous studies, prolonged exercise has been shown to impair $\mathrm{Ca}^{2+}$-ATPase activity and $\mathrm{Ca}^{2+}$ release and sequestration by the sarcoplasmic reticulum. However, the significance of alterations in $\mathrm{Ca}^{2+}$ cycling in relation to fatigue and the maintenance of homoeostasis in the peripheral physiological systems during exercise are not entirely clear. For example, a reduction in $\mathrm{Ca}^{2+}$ uptake, accompanied by a reduction in release of $\mathrm{Ca}^{2+}$, may be the result of a compensatory mechanism aimed at maintaining optimal intracellular $\mathrm{Ca}^{2+}$ concentrations. In addition, changes in $\mathrm{Ca}^{2+}$ cycling associated with fatigue may be secondary to alterations in the intracellular environment, including increased concentrations of $\mathrm{H}^{+}, \mathrm{P}_{\mathrm{i}}$, and ADP. This would be an example of a direct peripheral system modulator.

The interpretation of the role of $\mathrm{Ca}^{2+}$ cycling in the aetiology of fatigue during voluntary exercise remains difficult. In part, there are fibre-type specific factors that alter the $\mathrm{Ca}^{2+}$ dependent sequelae during muscular activity, as well as intensity and frequency dependent factors. However, it is also likely that intracellular $\mathrm{Ca}^{2+}$ concentrations and $\mathrm{Ca}^{2+}$ cycling may be influenced, at least in part, by feed forward control. For example, during voluntary muscle contraction, there is some evidence of a downregulation of muscle firing frequency and of rotation between motor units, thereby providing "protection" for the action potential and ATP-depletion, during so called maximal exercise. ${ }^{35}$

In summary, there is growing evidence that metabolic substrates and intermediates in muscle and elsewhere may act as signallers to the central nervous system. It appears that these signallers may provide crucial information on, for example, the energy status of the muscle cells to the central governor, allowing integration of this information with feed forward programming. Pacing strategy would thereby be altered as part of a non-linear, oscillating system for 
maintaining homoeostatic control of the peripheral physiological conditions.

\section{COMPLEX SYSTEM INTERACTION, NON-LINEAR MODELS, AND INTEGRATION OF FEED FORWARD AND FEEDBACK CONTROL OF METABOLIC ACTIVITY}

The central governor model of fatigue, incorporating teleoanticipation during exercise, is an example of complex, integrative control in which all physiological systems interact continuously in a deterministic manner. Were this system perfect, metabolite concentrations and physiological variables would remain constant during exercise, as any change would immediately activate afferent signals inducing a counterregulatory response, which would return the system to its baseline. However, all metabolic and physiological variables oscillate continuously at rest and during exercise. As mentioned previously, the amplitude of the change in a variable away from the baseline, and the extent and speed of its return to the baseline are known as the "gain" and time constant of the gain of the system. ${ }^{3}$ A perfect system with no changes away from baseline is described as having "infinite" gain. The success of homoeostatic control mechanisms in a physiological system is therefore directly related to the gain and the time constant of the gain of the different interacting metabolic and neural variables in the system.

Disease states, such as diabetes mellitus, result in alterations in the gain of the homoeostatic control of blood glucose concentrations. ${ }^{4}$ These changes result in greater oscillations of blood glucose concentrations with slower returns to baseline in response to imposed stresses. This change is caused by either reduced effectiveness of insulin on peripheral tissues or reduced production of insulin, or both, so that responses to changes in blood glucose concentrations are attenuated. ${ }^{36-38}$ Therefore a measure of a system's strength and fragility is its gain and the time constant of the gain. The greater the gain and the time constant of the gain, the more efficient are its physiological function and signalling processes.

During some forms of exercise, physiological variables such as heart rate oscillate continuously. ${ }^{39}$ This oscillation indicates that the gain of metabolic systems in the human system is not perfect. This effect is probably due to a lag between metabolic signalling and efferent feed forward neural adjustments. Moreover, physiological systems are not isolated, and are constantly being influenced by external factors from the environment, which may be unexpected and require adjustment from the preplanned, pacing strategies. These external factors may also result in a time related lag phase.

Another possible explanation for the oscillations and relatively modest gain control of metabolic variables may be that interactions occur between different peripheral systems continuously both at rest and during physical activity. The teleoanticipatory regulatory system of Ulmer ${ }^{5}$ describes a complex system of feed forward control of the motor unit recruitment, modified in some way by the interpretation of afferent feedback, arising from muscle (somatosensory) or other peripheral organs or both. This interpretation may be altered by factors such as training, muscle reserve, muscle metabolic rate, and prior or antecedent experiences, and the integration of feedback and feed forward control is continuous. Furthermore, interactions between different substrates and metabolites also occur in the periphery without direct central nervous system intervention.

An example of this integrative control is found in blood glucose kinetics. Blood glucose concentrations are directly affected by insulin concentrations, and blood insulin concentrations are directly affected in return by blood glucose concentrations.$^{40}$ If blood glucose concentrations fall, there is a concomitant fall in blood insulin concentrations, resulting in the reduction of the anabolic activity of enzymes which would convert glucose into less active forms of stored energy. The net effect is to increase blood glucose concentrations. However, as blood glucose concentrations increase, insulin concentrations also increase. This results in increased nonoxidative and oxidative disposal of glucose, thereby reducing blood glucose availability. Thus blood glucose concentrations oscillate with time, and these oscillations are caused directly by peripheral regulation rather than by a change in central feed forward command. ${ }^{36} 4041$ Similarly, insulin concentrations oscillate synchronously with blood glucose concentrations, although with a slight time delay. ${ }^{42}$ Therefore the changes in blood glucose and insulin concentrations are related in this model and are apparently independent of external control mechanisms.

The blood glucose/insulin control system appears to be self contained and well controlled. However, blood glucose concentrations are also affected by the concentrations of a number of other substrates and hormones in the peripheral metabolic system, as well as by neural activation, as described previously. For example, muscle and liver glycogen concentrations, blood lipid concentrations, and glucagon and catecholamine concentrations also all directly affect blood glucose concentrations. ${ }^{21}{ }^{37}$ These substances alter the simple relation between glucose and insulin concentrations by adding complexity to the interaction of the different components of the system. The more metabolic variables that directly affect blood glucose concentrations, the greater will be the stochastic nature of the oscillations in blood glucose concentrations, and the more likely that these fluctuations would appear to be random in nature. However, these apparently random fluctuations are shaped by the interaction of each different variable with the blood glucose concentration.

Furthermore, the blood glucose concentration itself affects a number of different peripheral and central physiological systems, and can cause changes in the function of these different systems. These lead to indirect alterations in blood glucose concentrations in a feed forward manner. For example, a decrease in blood glucose concentration would affect liver substrate metabolism, activating liver glycogenolysis to restore blood glucose concentrations. ${ }^{37}$ This process takes longer than the direct insulin and glucose interaction and would result in further adjustments of insulin and glucose concentrations. Decreases in blood glucose concentrations may also directly affect the brain regulatory centres and induce the release of hormones. The result is an increase in hepatic glucose output, and systemic glucose sparing, from the hypothalamic-pituitary-adrenal axis. Neuroglucopenia may also initiate cognitive behavioural strategies such as reduced motivation and drive to exercise. ${ }^{43}{ }^{44}$ These changes would then attenuate the efferent neural command, causing a reduction in skeletal muscle motor unit recruitment and power output, causing the metabolic rate to fall. The requirement for blood glucose as an energy fuel would consequently be lowered, thereby affecting the insulin and glucose interaction.

Therefore, at a number of functional levels and in different physiological systems, changes are initiated as a result of altering blood glucose concentrations, which induce multiple responses that indirectly affect the blood glucose concentrations in a feed forward manner. These indirect changes would result in an even more complex, seemingly stochastic oscillatory pattern to the control of blood glucose concentrations than would be the case in the isolated, peripherally based system (fig 3). During physical activity, the amplitude and frequency of these oscillations in blood glucose concentrations change, but the metabolic variables still "talk" to each other, and this interaction then becomes part of general 
homoeostasis. Alterations during physical activity appear to be designed to maximise the gain and the time constant of the gain at the particular metabolic rate, or to alter the metabolic rate as a consequence of the integrated system. Therefore it is possible that the final force output and resultant pacing strategy during physical activity also change in an oscillatory manner. ${ }^{23}{ }^{39}$ This is due, in part, to the continuous interactions between peripheral variables, and also the efferent motor command resulting from teleoanticipatory calculations, which is both a response to and a controller of these oscillations, and which ultimately contributes to the gain and the time constant of the gain of the system.

\section{SYSTEM REDUNDANCY AND HIERARCHICAL CONTROL MECHANISMS}

In this complex model of physiological regulation during exercise, the response to exercise involves the gathering of information from the periphery-for example, from the muscles, liver, and the blood composition-as well as centrally from the special senses-for example, vision, hearing - the internal clocks, and the emotional and memory systems. The central governor integrates these systems, in order to formulate calculations to control pacing strategy during exercise and responses to external perturbations. However, the changes in efferent neural command that the brain central governor initiates will also lead to alterations in peripheral metabolic activity which induce peripheral homoeostatic responses. These different levels create hierarchical control and a degree of system redundancy.

It is not clear why different levels of control are necessary, but this redundancy may provide more options for centrally integrated control of homoeostasis of the peripheral physiological systems in response to changes in the periphery. ${ }^{45}$ Thus the function of these redundant control systems may be to improve the gain and time constant of the gain of homoeostasis in the peripheral physiological systems. A complex system with more control choices may also be less brittle than a more simply controlled system. ${ }^{46}$ Pincus $^{46}$ suggested that the irregularity associated with multiple levels

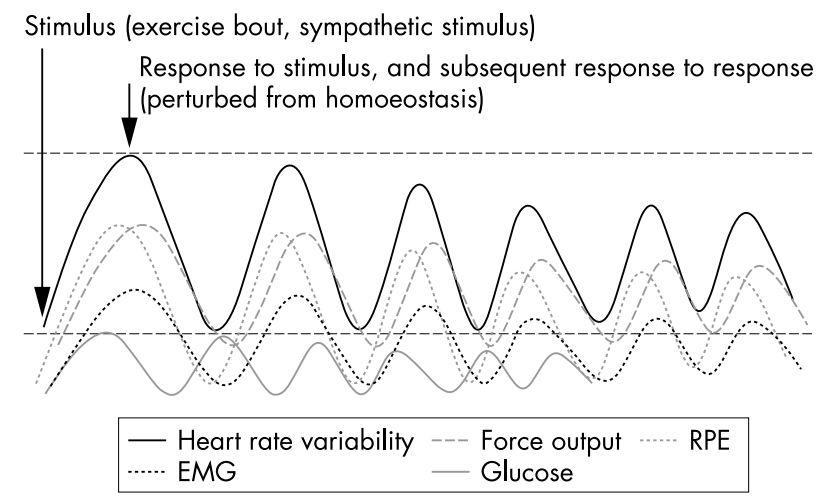

Figure 3 The exercise reflex and oscillating theory of control of physical activity. A stimulus such as exercise induces a response to the stimulus, and a subsequent response to this response at a later time point. This creates oscillatory activity of different physiological systems as part of homoeostatic control, with the aim of restoring baseline levels of activity and preventing catastrophic system failure. Amplitude and frequency of response can be monitored as an output signal from the periphery (infinite gain is like a flat line); it can be local (paracrine, autocrine) or distant (endocrine-neurotransmitter). An attenuation or amplification of response may be the result of some antecedent exposure, such as diet or training. Response adaptation may be chronic or acute-for example, catch up growth; micro-macro inter-relations between oscillations (timing, etc) may provide information. It may be functional or reflect dysfunction. RPE, Rating of perceived exertion; EMG, electromyography. of command processes and more complex systems are important for maintaining a healthy state, and that diseased systems become more isolated with fewer intersystem communications. A simpler system reduces the gain and time constant of the gain and makes physiological responses to perturbations less successful.

Therefore, paradoxically, the more complex the system, the more robust it may be. Redundancy and multiple feed forward and feedback control loops maintain homoeostatic levels of activity of a particular system, and do not allow the system to collapse completely if a single control mechanism fails. However, it is not clear whether some of these redundant control systems are more or less essential than others and whether, during routine activity, certain control mechanisms are used with greater frequency than others. Furthermore, not all control systems may be functionally redundant.

For example, Howlett et $a l^{47}$ and others ${ }^{48}$ have studied the humoral effects of adrenaline infusion, at concentrations similar or higher than those measured during moderate to high intensity exercise, during exercise of low to moderate intensity. Thus, adrenaline was infused at a rate similar to that experienced during high intensity exercise $(80 \%$ $\mathrm{VO}_{2}$ peak), while subjects exercised at a low intensity $(40 \%$ $\mathrm{VO}_{2}$ peak). This model therefore examined the effect of a humoral factor, typically activated by a neural stimulus, arising from increased exercise metabolic demand, but without the appropriate exercise intensity. They found that, although plasma adrenaline concentrations were the same in both conditions, hepatic glucose output was less than that measured during even short term exercise at $80 \% \mathrm{Vo}_{2}$ peak. This suggests that, although there is a potential redundancy, humoral control from the adrenal medulla may only be fully activated under conditions specific to efferent neural command and in relation to feedback from the muscle in terms of actual work output.

\section{SYSTEM MUTABILITY: EFFECT OF EXTERNAL PERTURBATION AND ANTECEDENT EXERCISE EXPOSURE ON CENTRAL NERVOUS SYSTEM REGULATION OF METABOLIC ACTIVITY}

The previous section described how a complex integrative control system maintains homoeostasis of the peripheral physiological systems during exercise through the interaction of multiple variables, and by interpreting both feed forward and feedback input, in a continuous and oscillating manner. It is not clear whether such a complex system is immutable or can be permanently altered by external perturbations or prior activity. However, recent work has shown that even a single bout of prior activity or a single episode of hypoglycaemia can alter and, in fact, attenuate the glucose counter-regulatory response during a subsequent intervention. For example, Davis et $a l^{49}$ conducted a trial in which healthy subjects were exposed to $2 \times 90$ minutes of either hypoglycaemia ( $\sim 2.9 \mathrm{mmol} / \mathrm{l}$ blood [glucose]) or euglycaemia. On the following day, subjects performed 90 minutes of cycling at $50 \% \mathrm{VO}_{2}$ peak, during which euglycaemia was maintained with an intravenous glucose infusion. Subjects who had been exposed to a single bout of antecedent hypoglycaemia had a completely attenuated counter-regulatory hormonal response, so that the expected rise in serum glucagon and adrenaline concentrations was noticeably blunted. As a consequence of this response, subjects required a 10-fold higher rate of glucose infusion to maintain euglycaemia during exercise. The teleological function of this response is not yet known. However, this antecedent exposure clearly changes the gain and time constant of the gain in the system and increases the complexity of integration of feed forward and feedback control. 


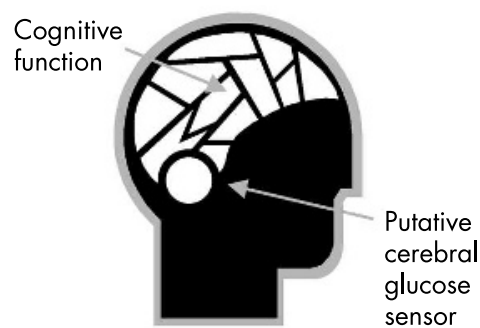

Euglycaemia

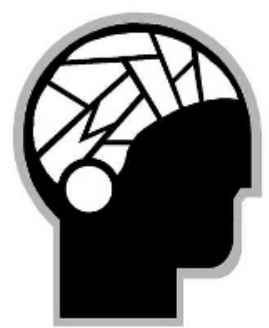

Hypoglycaemia + ketones

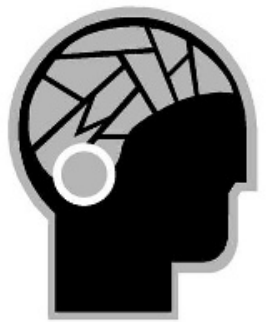

Hypoglycaemia

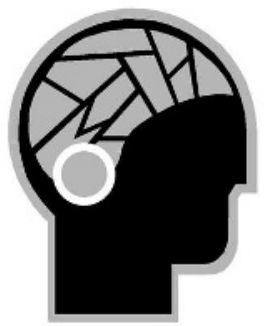

Hypoglycaemia + alanine

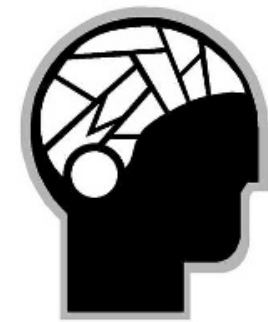

Hypoglycaemia + lactate

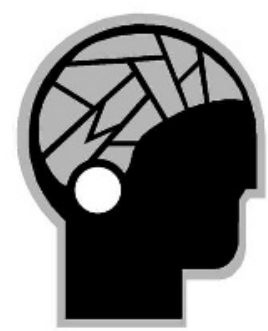

Hypoglycaemia + intralipid

Basal cerebral metabolic rate

Reduced cerebral metabolic rate

Figure 4 Differential sensitivity of regional brain function to hypoglycaemia. There are changes in cerebral metabolic rate of cognitive function or glucose sensor regions of the brain in response to hypoglycaemia and infusion of different metabolic fuels such as lactate, ketones, alanine, and intralipid. Reproduced, with permission, from Evans and Amiel. ${ }^{55}$

Chronic training also induces a number of changes to metabolic function in different physiological systems. ${ }^{50} 51$ These changes are probably due to protein regulatory changes which reset the gain and time constant of the gain of the different system interactions by altering the physical structures that control physiological activity. However, physical training does not induce permanent changes, as once the training stimulus is removed, the systems return to the original "detrained" homoeostatic set points of the different peripheral physiological systems. ${ }^{52}{ }^{53}$ Further work is needed to assess how the teleoanticipatory central control system changes in response to physiological perturbations such as training, illness, dietary manipulations, and activity at altitude, among many others.

\section{RELATION BETWEEN AFFERENT INPUT OF METABOLIC ACTIVITY AND THE CONSCIOUS PERCEPTION OF THE SENSATION OF FATIGUE}

The relation between this afferent input from peripheral metabolic activity and the conscious perception of the sensation of fatigue is also complex. When individuals are subjected to a single bout of antecedent hypoglycaemia, not only is the metabolic counter-regulatory response attenuated, but "hypoglycaemia unawareness" develops, in which there is reduced conscious perception of the symptoms associated

\section{What is already known on this topic}

Previous work on fatigue has examined each physiological variable as a discrete entity, which has led to a reductionist approach to understanding fatigue. In these models, changes in peripheral systems such as substrate depletion or metabolite accumulation are described as direct causes of fatigue. with hypoglycaemia. Therefore downregulation of the perceptual processes must occur in the brain centres responsible for the perception of low blood glucose concentrations. At present, the exact brain site and brain processes involved in the conscious perception of fatigue and hypoglycaemia have not been established. ${ }^{54}$

The conscious perception of hypoglycaemia has been studied by Evans and Amiel. ${ }^{55}$ Using positron emission tomography of the brain, they studied regional glucose uptake and metabolism, as well as cognitive function, under different experimental conditions. They found that there was differential regulation of metabolism in different brain regions, and that physical control function could be maintained under conditions of hypoglycaemia in conjunction with lipid infusion, but cognitive function was impaired under these conditions (fig 4). In contrast, the provision of lactate and ketones maintained control of both cognitive and physical function by the brain. They speculated that hypoglycaemia is detected by the brain, possibly as a result of the fall in metabolic rate in sensitive areas of the ventromedial hypothalamus, and thus alternative fuel supplies may be used to maintain cognitive function.

Recently, we ${ }^{56}$ conducted a study which provided an intriguing caveat to these studies. After three days of

\section{What this article adds}

In this article, fatigue is examined as being the result of the complex interaction of multiple peripheral physiological systems and the brain. In this new model, all changes in peripheral physiological systems such as substrate depletion and metabolite accumulation act as afferent signallers which modulate control processes in the brain in a dynamic, nonlinear, integrative manner. 
following a low carbohydrate diet, well trained cyclists exercised for 150 minutes at $70 \% \mathrm{Vo}_{2}$ peak while receiving either intravenous glucose to maintain euglycaemia or placebo saline infusion. Not unexpectedly, glucose infusion increased the exercise time to fatigue. However, this may not have occurred as a direct result of the reversal of hypoglycaemia, as the subjects who exercised for the longest with the placebo infusion, still reached the lowest blood glucose concentrations. Two subjects were clinically hypoglycaemic, yet had the greatest counter-regulatory response. The results suggest that perception of fatigue may be altered by changes in blood glucose concentration, but that there is an individual or differential sensitivity and perception of hypoglycaemia, perhaps based on antecedent exposures.

Furthermore, there may be conditions that make the interpretation of the effect of blood glucose concentrations on both performance and RPE more complex. For example, Clark et $a l^{57}$ found that subjects ingesting a carbohydrate drink during a one hour time trial, and who were told that they were ingesting carbohydrate, performed significantly better than those receiving carbohydrate but were misinformed that they were receiving placebo. Even more surprisingly, subjects receiving a placebo, who were truthfully informed, performed significantly better during the time trial than did those who received carbohydrate, but who were misinformed that they were ingesting placebo. This study highlights the complexity of the interaction between motivation, expectation, and performance, and provides further evidence for the integrative teleoanticipatory model of exercise performance developed in this series of papers.

\section{SUMMARY}

In the fable of the blind man and the elephant, we see illustrated a reductionist approach, in which the interpretation of the individual parts are made without consideration of the system as a whole. In the context of a complex and integrated model of fatigue during exercise, there is no single regulatory component. Rather, there are multiple levels of regulation, resulting in integrative homoeostatic control of the different physiological systems, which is a continuous and oscillatory process, and which thereby invokes compensatory responses in the periphery. There is also, however, evidence for central programming and a feed forward component in this complex model, which serves to alter the settings at which gain and the time constant of the gain of the entire system are achieved, and which thereby alters the perception of fatigue.

Furthermore, in this complex model, there are redundancies in control mechanisms that can be altered by antecedent exposures to exercise or metabolic perturbations and external environmental factors. These multiple controls and redundancies produce a robust system, providing for changes in gain and the time constant of the gain as part of a general homoeostatic process. These homoeostatic controls maintain system integrity and ensure that any system is not overwhelmed or used to absolute maximal capacity using continuous feed forward and feedback control. The integrated complex governor model of fatigue therefore recognises multiple interdependent control systems, the importance of peripheral metabolic factors as signallers, and that the teleoanticipatory central nervous system is mutable and can be "reset" or "hard wired" by various stimuli such as prior experience, antecedent exposures, and training. Finally, in the development of any biological model, it is useful to consider or acknowledge the underlying teleological framework of the evidence on which the model is advanced, in order to fully understand its potential limitations.
Authors' affiliations

E V Lambert, A St Clair Gibson, T D Noakes, Research Unit for Exercise Science and Sports Medicine, University of Cape Town, Newlands, South Africa

Conflict of interests: none declared

\section{REFERENCES}

1 Noakes TD, St Clair Gibson A. Logical limitations in the "catastrophe" models of fatigue during exercise in humans. Br J Sports Med 2004;38.

2 St Clair Gibson A, Noakes TD. Evidence for complex system integration and dynamic neural regulation of skeletal muscle recruitment during exercise in humans. Br J Sports Med 2004;38

3 Koeslag JH, Saunders PT, Wessels JA. Glucose homoeostasis with infinite gain: further lessons from the Daisyworld parable? J Endocrinol 1997; 154:187-92.

4 Koeslag JH, Saunders PT, Wessels JA. The chromogranins and the counter regulatory hormones: doe they make homoeostatic sense? J Physiol 1999;517:643-9.

5 Ulmer H-V. Concept of an extracellular regulation of muscular metabolic rate during heavy exercise in humans by psychophysiological feedback. Experientia 1996;52:416-20.

6 Hampson DB, St Clair Gibson A, Lambert Ml, et al. Effects of deception on perceived exertion during high-intensity running bouts. Percept Mot Skills 2003;98:1027-38.

7 Hampson DB, St Clair Gibson A, Lambert MI, et al. The influence of sensory cues on the perception of effort during exercise and central regulation of exercise performance. Sports Med 2001;31:935-52.

8 Nybo L, Nielsen B. Perceived exertion is associated with an altered brain activity during exercise with progressive hyperthermia. J Appl Physiol 2001;91:2017-23.

9 Kvist A, Lindstrom A, Green M, et al. Carrying large fuel loads during sustained bird flight is cheaper than expected. Nature 2001;413:730-2.

10 Jenni L, Jenni-Eiermann S, Spina F, et al. Regulation of protein breakdown and adrenocortical response to stress in birds during migratory flight. Am J Physiol 2000;278:R1182-9.

11 Vissing J, Iwamoto GA, Rybicki KJ, et al. Mobilization of glucoregulatory hormones and glucose by hypothalamic locomotor centers. Am J Physiol 1989;257:E722-8.

12 Vissing J, Wallace JL, Scheurink AJW, et al. Ventromedial hypothalamic regulation of hormonal and metabolic responses to exercise. Am J Physiol 1989;256:R1019-26.

13 Vissing J, Andersen M, Diemer NH. Exercise-induced changes in local cerebral glucose utilization in the rat. J Cereb Blood Flow Metab 1996;16:729-36.

14 Vissing J. Muscle reflex and central motor control of neuroendocrine activity, glucose homoeostasis and circulation during exercise. Acta Physiol Scand (Suppl) 2000;647: 1-26.

15 van Dijk G, Vissing J, Steffens $A B$, et al. Effect of anaesthetizing the region of the paraventricular hypothalamic nuclei on energy metabolism during exercise in the rat. Acta Physiol Scand 1994;151:165-72.

16 Seals DR, Enoka RM. Sympathetics activation is associated with increases in EMG during fatiguing exercise. J Appl Physiol 1989;66:88-95.

17 Ray CA, Mark AL. Sympathetic nerve activity to non-active muscle of the exercising and non-exercising limb. Med Sci Sports Exerc 1995;27:183-7.

18 Victor RG, Secher NH, Lyson T, et al. Central command increases muscle sympathetic nerve activity during intense intermittent isometric exercise in humans. Circ Res 1995;76:127-31.

19 Ray CA. Sympathetic adaptations to one-legged training. J Appl Physiol 1999;86: 1583-7.

20 Hunter SK, Enoka R. Changes in muscle activation can prolong the endurance time of a submaximal isometric contraction in humans. J Appl Physiol 2002;94:108-18

21 Weltan SM, Bosch AN, Dennis SC, et al. Influence of muscle glycogen content on metabolic regulation. Am J Physiol 1998;274:E72-82.

22 Rauch LH, Rodger I, Wilson GR, et al. The effects of carbohydrate loading on muscle glycogen content and cycling performance. Int J Sports Nutr 1995;5:25-36.

23 Kay D, Marino FE, Cannon J, et al. Evidence for neuromuscular fatigue during high intensity exercise in warm humid conditions. Eur J Appl Physiol 2001;84:115-21.

24 Noakes TD. Commentary to accompany: Training and bioenergetic characteristics in elite male and female Kenyan runners. Med Sci Sports Exerc 2003;35:305-6.

25 Polekhina G, Gupta A, Mitchell BJ, et al. AMPK beta subunit targets metabolic stress sensing to glycogen. Curr Biol 2003; 13:867-71.

26 Stephenson DG, Nguyen LT, Stephenson GM. Glycogen content and excitation-contraction coupling in mechanically skinned muscle fibres of the cane toad. J Physiol 1999 15;519:177-87.

27 Steensberg A, van Hall G, Keller C, et al. Muscle glycogen content and glucose uptake during exercise in humans: influence of prior exercise and dietary manipulation. J Physiol 2002 15;541:273-81.

28 Brooks GA. Current concepts in lactate exchange. Med Sci Sports Exerc $1991 ; 23: 895-906$

29 Nielsen OB, de Paoli F, Overgaard K. Protective effect of lactic acid on force production in rat skeletal muscle. J Physiol 2001;536:161-6. 
30 Immke DC, McCleskey EW. Lactate enhances the acid-sensing $\mathrm{Na}^{+}$channel on ischemia-sensing neurons. Nat Neurosci 2001;4:869-70.

31 Dalsgaard MK, Nybo L, Cai Y, et al. Cerebral metabolism is influenced by muscle ischaemia during exercise in humans. Exp Physiol 2003;88:297-302.

32 Smith LL. Cytokine hypothesis of overtraining: a physiological adaptation to excessive stress. Med Sci Sports Exerc 2000;32:317-31.

33 Jones DA, Round JM. Skeletal muscle in health and disease. Manchester: Manchester University Press, 1990.

34 Green HJ. Adaptations in the muscle cell to training: role of the $\mathrm{Na}^{+}-\mathrm{K}^{+}$ ATPase. Can J Appl Physiol 2003;25:204-16

35 Green HJ. Muscular factors in endurance. In: Shephard RJ, Astrand PO, eds. Endurance in sport. London: Blackwell Science LTD, 2000:158-83.

36 Hollingdal M, Juhl CB, Pincus SM, et al. Failure of physiological plasma glucose excursions to entrain high-frequency pulsatile insulin secretion in type 2 diabetes. Diabetes 2000;49:1334-40.

37 Khan AH, Pessin JE. Insulin regulation of glucose uptake: a complex interplay of intracellular signaling pathways. Diabetologia 2002;45:1475-83.

38 Simon C, Weibel L, Brandenberger G. Twenty-four-hour rhythms of plasma glucose and insulin secretion rate in regular night workers. Am J Physiol 2000;278:E413-20.

39 Palmer GS, Hawley JA, Dennis SC, et al. Heart rate responses during a 4-d cycle race. Med Sci Sports Exerc 1994;26:1278-83.

40 Saunders PT, Koeslag JH, Wessels JA. Integral rein control in physiology. J Theor Biol 1998;194:163-73.

41 Sturis J, Van Cauter E, Blackman JD, et al. Entrainment of pulsatile insulin secretion by oscillatory glucose infusion. J Clin Invest 1991;87:439-45.

42 Goodner CJ, Walike BC, Koerker DJ, et al. Insulin, glucagon, and glucose exhibit synchronous, sustained oscillations in fasting monkeys. Nature 1977; 195:177-9.

43 Smith D, Amiel SA. Hypoglycaemia unawareness and the brain. Diabetologia 2002;45:949-58
44 Smith D, Pernet A, Reid $\mathrm{H}$ et al. The role of hepatic portal glucose sensing in modulating responses to hypoglycaemia in man. Diabetologia 2002;45: 1416-24.

45 Latash ML, Anson JG. What are "normal movements" in atypical populations? Behav Brain Sci 1996;19:55-106.

46 Pincus SM. Greater signal regularity may indicate increased system isolation. Math Biosci 1994;122:161-81.

47 Howlett K, Febbraio M, Hargreaves M. Glucose production during strenuous exercise in humans: role of epinephrine. Am J Physiol 1999;276:E1130-5.

48 Moira-Rodriguez R, Coyle EF. Effects of plasma epinephrine on fat metabolism during exercise: interactions with exercise intensity. Am J Physiol Endocrinol Metab 2000;278:E669-76.

49 Davis SN, Galassetti P, Wasserman DH, et al. Effects of antecedent hypoglycaemia on subsequent counterregulatory responses to exercise. Diabetes 2000;49:73-81.

50 Deschenes MR, Kraemer WJ. Performance and physiological adaptations to resistance training. Am J Phys Med Rehabil 2002;81:S3-16.

51 Noakes TD. Lore of running. Cape Town: Oxford University Press, 2001.

52 Mujika I, Padilla S. Cardiorespiratory and metabolic characteristics of detraining in humans. Med Sci Sports Exerc 2001;33:413-21.

53 Mujika I, Padilla S. Muscular characteristics of detraining in humans. Med Sci Sports Exerc 2001;33:1297-303.

54 St Clair Gibson A, Baden DA, Lambert Ml, et al. The conscious perception of the sensation of fatigue. Sports Med 2003;33:167-76.

55 Evans M, Amiel S. Carbohydrates as a cerebral metabolic fuel. J Pediatr Endocrinol Metab 1998;11:99-102.

56 Claassen A, Noakes TD, Lambert EV, et al. Evidence of differential sensitivity to hypoglycaemia in carbohydrate-depleted subjects. Med Sci Sports Exerc 2001;33(suppl 5):S154.

57 Clark VR, Hopkins WG, Hawley JA, et al. Placebo effect of carbohydrate feedings during a $40-\mathrm{km}$ cycling time trial. Med Sci Sports Exerc 2000;32: 1642-7. 